\title{
Ivacaftor in a young boy with the rare gating mutation S549R - use of lung clearance index to track progress: a case report
}

Nina Lenherr ${ }^{\dagger}$, Marco Lurà ${ }^{\dagger}$, Daniel Trachsel, Philipp Latzin and Juerg Hammer ${ }^{*}$

\begin{abstract}
Background: Ivacaftor acts as a potentiator of the cystic fibrosis transmembrane conductance regulator (CFTR) and increases the transepithelial chloride transport of CFTR in 9 of 10 known gating mutations causing cystic fibrosis. S549R is a rare gating mutation considered to be less sensitive to potentiators than all other gating mutations.

Case presentation: We report our first experience with ivacaftor in an 8-year-old boy with the rare S549R gating mutation. Besides subjective clinical improvements, the sweat chloride level and the lung clearance index decreased impressively within a few weeks of treatment while forced expiratory volume in the first second values remained in normal range.

Conclusion: We emphasize the value of measuring small airway function by lung clearance index as an outcome measure for new interventions targeting the correction of the CFTR defect at an age before traditional lung function parameters start to deteriorate.
\end{abstract}

Keywords: Cystic fibrosis, Ivacaftor, S549R, Gating mutation, $\mathrm{N}_{2} \mathrm{MBW}$, LCl

\section{Background}

Ivacaftor acts as a potentiator of the cystic fibrosis transmembrane conductance regulator (CFTR) and increases the transepithelial chloride $\left(\mathrm{Cl}^{-}\right)$transport of CFTR in 9 of 10 known gating mutations and in $\mathrm{R} 117 \mathrm{H}$ mutation (class IV) causing cystic fibrosis. Ivacaftor has been approved in 2012 by the US Food and Drug Administration (FDA) for G551D, the most common gating mutation, after improvements in lung function and lowering of sweat chloride levels had been demonstrated in clinical trials [1]. In 2014 the approval was extended for 8 additional gating mutations, including S549R. S549R is a rare gating mutation (41 described patients in CFTR2 [2]) primarily described in the Bedouin population of the United Arab Emirates, in Saudi Arabia and in North Africa. The clinical phenotype seen with the homozygous S549R mutation is generally severe and similar to homozygous deltaF508 mutation [3, 4]. This mutation causes an additional

\footnotetext{
* Correspondence: juerg.hammer@ukbb.ch

${ }^{\dagger}$ Equal contributors

Division of Intensive Care and Pulmonology, University Children's Hospital Basel (UKBB), University of Basel, Spitalstrasse 33, CH-4056 Basel, Switzerland
}

mild processing defect besides the defective CFTR channel gating resulting in a lower in vitro response to ivacaftor than all the other gating mutations [5]. Information on clinical benefits of ivacaftor in this particularly rare mutation is scarce at present [6].

The measurement of ventilation inhomogeneity, such as the lung clearance index (LCI), is more sensitive than forced expiratory volume in the first second (FEV1) to detect early lung function abnormalities [7-10]. Further, normal tidal breathing is often easier to perform for younger children than forced expiratory maneuvers. LCI, measured by $\mathrm{N}_{2}$ multiple-breath washout $\left(\mathrm{N}_{2} \mathrm{MBW}\right)$, represents the number of lung volume turnovers required to clear the lung of $\mathrm{N}_{2}$ to $1 / 40^{\text {th }}$ of the starting concentration [10]. The duration of the test depends on the degree of ventilation inhomogeneity and can therefore be time-consuming in very severe lung disease. We report our first experience with ivacaftor and the usefulness of LCI in a young Swiss patient with the S549R mutation to demonstrate improvements in lung function in response to this therapy when FEV1 is within normal limits. 


\section{Case presentation Case report}

An 8-year-old boy with S549R/1717-1G > A genotype was started on ivacaftor (150 mg b.i.d.) on compassionate use. At the age of 9 months he was diagnosed with CF due to failure to thrive. His previous history was remarkable for recurring nasal polyposis requiring endoscopic surgery and exocrine pancreatic insufficiency necessitating enzyme replacement therapy. He grew with body weight and height along the $10^{\text {th }}$ percentile. He suffered from rather mild respiratory symptoms, primarily intermittent productive cough, and had close to normal lung function parameters in previous years as measured by body plethysmography and spirometry (minimal z-score of FEV1: -1.2). Sputum cultures grew Haemophilus influenzae and Staphylococcus aureus on several occasions. After 6 weeks of ivacaftor treatment, the patient reported clinical improvements in cough frequency, sputum production, physical performance, and less salt cravings. He gained $1.4 \mathrm{~kg}$ in body weight without changing the dose of his pancreatic enzyme replacement therapy. His sweat chloride level (Macroduct ${ }^{\circ}$ ) decreased from $115 \mathrm{mmol} / \mathrm{l}$ before ivacaftor to $40 \mathrm{mmol} / \mathrm{l}$ after 6 weeks and $52 \mathrm{mmol} / \mathrm{l}$ after 41 weeks (normal $<30 \mathrm{mmol} / \mathrm{l}$ [11]) of treatment. His FEV1 increased from $1.25 \mathrm{~L}$ (-1.2 z-score) to $1.65 \mathrm{~L}$ (+0.5 z-score) after 41 weeks of ivacaftor therapy. The LCI (normal $<8$ ) measured by $\mathrm{N}_{2}-\mathrm{MBW}$ decreased from 14.5 to 8.3 after 6 weeks and 7.8 after 41 weeks of ivacaftor treatment (Table 1 and Fig. 1).

\section{Discussion}

Ivacaftor is the first authorized drug that improves defective CFTR function in rare mutations by potentiating the CFTR channel gating function. In vitro data suggests that ivacaftor has a similar effect on 9 of 10 CFTR gating mutations. However, the weakest drug effect was described

Table 1 Improvement of functional parameters during ivacaftor therapy

\begin{tabular}{|c|c|c|c|c|c|}
\hline & Start & 6 weeks & 12 weeks & 28 weeks & 41 weeks \\
\hline $\begin{array}{l}\text { Sweat chloride level } \\
{[\mathrm{mmol} / \mathrm{l}]}\end{array}$ & 115 & 40 & 30 & 40 & 52 \\
\hline FEV1 [l ] (z-score) ${ }^{a}$ & $\begin{array}{l}1.25 \\
(-1.2)\end{array}$ & $\begin{array}{l}1.53 \\
(0.5)\end{array}$ & $\begin{array}{l}1.48 \\
(0.2)\end{array}$ & $\begin{array}{l}1.63 \\
(0.7)\end{array}$ & $\begin{array}{l}1.65 \\
(0.5)\end{array}$ \\
\hline $\mathrm{LCl}$ (z-score) ${ }^{b}$ & $\begin{array}{l}14.5 \\
(14.2)\end{array}$ & $\begin{array}{l}8.3 \\
(2.4)\end{array}$ & $\begin{array}{l}7.6 \\
(1.1)\end{array}$ & $\begin{array}{l}8.1 \\
(2.0)\end{array}$ & $\begin{array}{l}7.8 \\
(1.5)\end{array}$ \\
\hline $\begin{array}{l}\text { Weight [kg] } \\
\text { (z-score) }\end{array}$ & $\begin{array}{l}21.8 \\
(-1.5)\end{array}$ & $\begin{array}{l}23.2 \\
(-1.1)\end{array}$ & $\begin{array}{l}23.7 \\
(-0.9)\end{array}$ & $\begin{array}{l}23.9 \\
(-0.9)\end{array}$ & $\begin{array}{l}26 \\
(-0.5)\end{array}$ \\
\hline $\begin{array}{l}\text { BMI }\left[\mathrm{kg} / \mathrm{m}^{2}\right] \\
\text { (z-score) }\end{array}$ & $\begin{array}{l}14.6 \\
(-0.8)\end{array}$ & $\begin{array}{l}15.6 \\
(-0.2)\end{array}$ & $\begin{array}{l}15.7 \\
(-0.1)\end{array}$ & $\begin{array}{l}15.5 \\
(-0.2)\end{array}$ & $\begin{array}{l}16.4 \\
(0.2)\end{array}$ \\
\hline
\end{tabular}

Improvement of different functional parameters during 41 weeks of ivacaftor therapy: FEV1 Forced expiratory volume in the first second, $L C l$ lung clearance index. ${ }^{\text {a }}$ Reference population for $z$-score values of FEV1 are the global multiethnic reference equations of the global lung function initiative [16]. ${ }^{b}$ Reference population for $\mathrm{z}$-score values of the $\mathrm{LCl}$ is our intracentric age-matched healthy control population

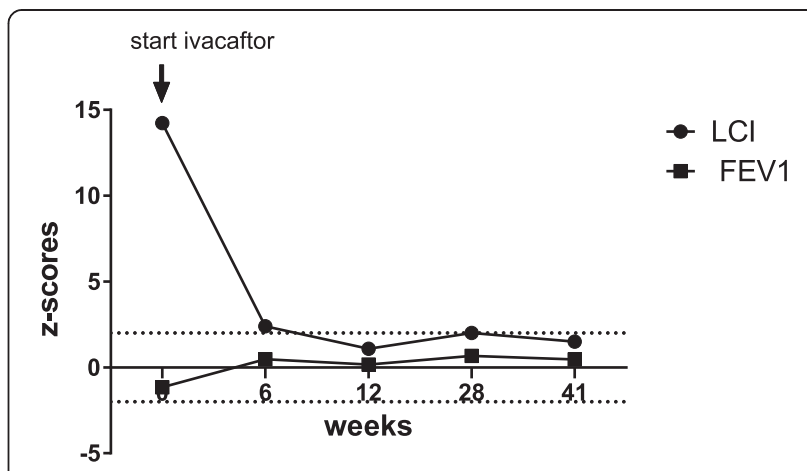

Fig. 1 Changes in z-score of $\mathrm{LCl}$ and FEV1 during ivacaftor treatment

for the S549R mutation [5]. S549R was initially considered a class II mutation leading to defective CFTR protein processing [12]. Recent electrophysiological studies using Fischer rat thyroid cells have suggested the presence of a predominant gating defect besides a mild processing defect of CFTR in S549R [5]. The processing defect may account for the lower ivacaftor response observed in vitro. Against this background, the impressive clinical and functional improvement in our patient with the S549R mutation was better than expected. These results are in line with a recently published clinical trial study including four patients with S549R mutation [6].

One limitation of this report is that no nasal potential differences or intestinal current measurement have been performed as further functional parameters. The benefit of using LCI as an outcome measure should not be over generalised from this case. However this report highlights the additional value of LCI as a sensitive parameter in relation to the traditional lung function parameter FEV1. Other studies have shown that the LCI of $\mathrm{N}_{2} \mathrm{MBW}$ is particularly useful in monitoring the early course of lung disease in young children with CF, particularly in those with normal spirometry $[13,14]$. The LCI is already elevated in presymptomatic or minimally symptomatic infants and young children with CF [9]. In our patient, the LCI decreased after 41 weeks of ivacaftor treatment from a z-score of 14.2 to 1.5 , while the z-score for FEV1 remained within the normal range ( $\mathrm{z}$-score -1.2 to 0.5 ). This is in agreement with previous work indicating that LCI is a more sensitive parameter to detect treatment success in young CF patients compared to spirometry, especially in children with little respiratory symptoms and near-normal spirometric lung volumes such as FEV1 [15].

\section{Conclusion}

This report provides anecdotal evidence of benefit of ivacafor in S549R mutation.

Further it illustrates the potential value of lung clearance index to serve as an outcome measure for new interventions targeting the correction of the CFTR defect 
at an early stage of the disease. This is relevant since ivacaftor approval has recently been extended to preschool children where performance and interpretation of spirometry is even more challenging. Such measurements may help to convince medical healthcare payers to cover the cost of the drug in our young CF population.

\section{Consent}

Written informed consent was obtained from the parents of the patient for publication of this case report and any accompanying images. A copy of the written consent is available for review by the Editor of this journal.

\section{Abbreviations}

CFTR: Cystic fibrosis transmembrane conductance regulator; $\mathrm{Cl}^{-}$: Chloride; FDA: Food and drug administration; FEV1: Forced expiratory volume in the first second; LCl: Lung clearance index; $\mathrm{N}_{2} \mathrm{MBW}$ : Nitrogen multiple-breath washout.

\section{Competing interests}

The authors declare that they have no competing interests.

\section{Authors' contributions}

$\mathrm{NL}$ and ML contributed equally to this article. All authors contributed to this case report and all authors read and approved the final manuscript.

\section{Acknowledgements}

The authors would like to thank the patient and his parents for the informed consent to this report.

Received: 28 April 2015 Accepted: 8 October 2015

Published online: 16 October 2015

\section{References}

1. Davies JC, Wainwright CE, Canny GJ, Chilvers MA, Howenstine MS, Munck A, et al. Efficacy and safety of ivacaftor in patients aged 6 to 11 years with cystic fibrosis with a G551D mutation. Am J Respir Crit Care Med. 2013;187(11):1219-25.

2. Castellani C, Team C. CFTR2: how will it help care? Paediatr Respir Rev. 2013;14 Suppl 1:2-5

3. Frossard PM, Hertecant J, Bossaert Y, Dawson KP. Genotype-phenotype correlations in cystic fibrosis: clinical severity of mutation S549R(T-G). Eur Respir J. 1999;13(1):100-2.

4. Dawson KP, Frossard PM, Al-Awar B. Disease severity associated with cystic fibrosis mutations deltaF508 and S549R(T-G). East Mediterr Health J. 2001;7(6):975-80.

5. Yu H, Burton B, Huang C-J, Worley J, Cao D, Johnson Jr JP, et al. Ivacaftor potentiation of multiple CFTR channels with gating mutations. J Cyst Fibros. 2012;11(3):237-45.

6. De Boeck K, Munck A, Walker S, Faro A, Hiatt P, Gilmartin G, et al. Efficacy and safety of ivacaftor in patients with cystic fibrosis and a non-G551D gating mutation. J Cyst Fibros. 2014;13(6):674-80.

7. Singer F, Kieninger E, Abbas C, Yammine S, Fuchs $O$, Proietti $E$, et al. Practicability of nitrogen multiple-breath washout measurements in a pediatric cystic fibrosis outpatient setting. Pediatr Pulmonol. 2013;48(8):739-46.

8. Kraemer R, Blum A, Schibler A, Ammann RA, Gallati S. Ventilation inhomogeneities in relation to standard lung function in patients with cystic fibrosis. Am J Respir Crit Care Med. 2005;171(4):371-8.

9. Aurora P, Bush A, Gustafsson P, Oliver C, Wallis C, Price J, et al. Multiplebreath washout as a marker of lung disease in preschool children with cystic fibrosis. Am J Respir Crit Care Med. 2005;171(3):249-56.

10. Robinson PD, Latzin P, Verbanck S, Hall GL, Horsley A, Gappa M, et al. Consensus statement for inert gas washout measurement using multiple- and single- breath tests. Eur Respir J. 2013;41(3):507-22.

11. De Boeck K, Wilschanski M, Castellani C, Taylor C, Cuppens H, Dodge J, et al. Cystic fibrosis: terminology and diagnostic algorithms. Thorax. 2006;61(7):627-35.
12. Welsh MJ, Smith AE. Molecular mechanisms of CFTR chloride channel dysfunction in cystic fibrosis. Cell. 1993;73(7):1251-4.

13. Kent $L$, Reix $P$, Innes JA, Zielen S, Le Bourgeois M, Braggion C, et al. Lung clearance index: evidence for use in clinical trials in cystic fibrosis. J Cyst Fibros. 2014;13(2):123-38.

14. Vanderhelst E, De Meirleir L, Schuermans D, Malfroot A, Vincken W, Verbanck S. The lung clearance index as a probe for the effectiveness of short-term therapies in cystic fibrosis lung disease. J Cyst Fibros. 2015;14(2):285-6.

15. Davies J, Sheridan H, Bell N, Cunningham S, Davis SD, Elborn JS, et al. Assessment of clinical response to ivacaftor with lung clearance index in cystic fibrosis patients with a G551D-CFTR mutation and preserved spirometry: a randomised controlled trial. Lancet Respir Med. 2013;1(8):630-8.

16. Quanjer PH, Stanojevic S, Cole TJ, Baur X, Hall GL, Culver BH, et al. Multi-ethnic reference values for spirometry for the 3-95-yr age range: the global lung function 2012 equations. Eur Respir J. 2012;40(6):1324-43.

\section{Submit your next manuscript to BioMed Central and take full advantage of:}

- Convenient online submission

- Thorough peer review

- No space constraints or color figure charges

- Immediate publication on acceptance

- Inclusion in PubMed, CAS, Scopus and Google Scholar

- Research which is freely available for redistribution 